\title{
The rigidity of three flavor quark matter
}

\author{
Massimo Mannarelli* \\ Instituto de Ciencias del Espacio (IEEC/CSIC) Campus Universitat Autònoma de Barcelona, \\ Facultat de Ciències, Torre C5, E-08193 Bellaterra (Barcelona), Spain \\ E-mail: massimodieec.uab.es
}

\section{Rishi Sharma ${ }^{\text {th }}$}

Los Alamos National Laboratory

E-mail: rishielanl.gov

Cold three flavor quark matter at large (but not asymptotically large) densities may exist in a crystalline color superconducting phase. These phases are characterized by a gap parameter $\Delta$ that varies periodically in space, forming a crystal structure. A Ginzburg-Landau expansion in $\Delta$ shows that two crystal structures based on cubic symetry are particularly favorable, and may be the ground state of matter at densities present in neutron star cores. We derive the effective action for the phonon fields that describe space- and time-dependent fluctuations of the crystal structure formed by $\Delta$, and obtain the shear modulus from the coefficients of the spatial derivative terms. Within a Ginzburg-Landau approximation, we find shear moduli which are 20 to 1000 times larger than those of neutron star crusts. This phase of matter is thus more rigid than any known material in the universe, but at the same time the crystalline color superconducting phase is also superfluid. These properties raise the possibility that the presence of this phase within neutron stars may have distinct implications for their phenomenology. For example, (some) pulsar glitches may originate in crystalline superconducting neutron star cores.

8th Conference Quark Confinement and the Hadron Spectrum

September 1-6, 2008

Mainz. Germany

\footnotetext{
${ }^{*}$ Speaker.

$\dagger$ Speaker.

†Joint contribution of Massimo Mannarelli and Rishi Sharma. We thank Krishna Rajagopal for collaboration
} 


\section{Introduction}

At the extreme densities present at the cores of the neutron stars, quarks may be deconfined. At present, the most promising direction to resolve whether quark matter is present in these compact objects is to find consequences of its presence for their phenomenology, which may allow observations to rule out or in this possibility.

According with qantum-chromodynamics (QCD), quark-quark interaction is strong and attractive between quarks that are antisymmetric in color, so we expect cold dense quark matter to be in a color superconducting state. The critical temperatures of these phases are generically on the order of tens of $\mathrm{MeV}$, much larger than the tens of $\mathrm{keV}$ temperatures of neutron star cores [1]. This implies that if quark matter is present there, it must be in a color superconducting phase, and also allows us to work at temperature $T=0$ for the calculations we discuss below.

At asymptotic densities, where the up $(u)$, down $(d)$ and strange $(s)$ quarks can all be treated as massless, quark matter is in the Color Flavor Locked (CFL) phase [2, 1]. The CFL condensate is antisymmetric in color and flavor indices and therefore involves pairing between quarks of different colors and flavors. All fermionic excitations are gapped, with a gap parameter $\Delta_{0} \sim 10-100 \mathrm{MeV}$.

However, at the cores of neutron stars, the quark number chemical potential $\mu$ is expected to be between 350 and $500 \mathrm{MeV}$ which means that $M_{s}$, lying somewhere between its current mass of order $100 \mathrm{MeV}$ and its vacuum constituent mass of order $500 \mathrm{MeV}$, cannot be neglected. In addition bulk matter must be in weak equilibrium and must be electrically and color neutral. These factors tend to separate the quark Fermi surfaces by $\sim M_{s}^{2} / \mu$, and thus disfavor the cross-species BCS pairing which characterizes the CFL phase. In neutral unpaired quark matter in weak equilibrium, to lowest order in $M_{s}^{2} / \mu^{2}$, the quarks can be treated as if they were massless, but with chemical potential splittings $\delta \mu_{2} \equiv\left(\mu_{u}-\mu_{s}\right) / 2$ and $\delta \mu_{3} \equiv\left(\mu_{d}-\mu_{u}\right) / 2$ given by $\delta \mu_{2}=\delta \mu_{3} \equiv \delta \mu=M_{s}^{2} /(8 \mu)$. Note that the splitting between unpaired Fermi surfaces increases with decreasing density. In the CFL phase, the Fermi momenta are not given by these optimal values for unpaired quark matter; instead, the system pays a free energy price $\propto \delta \mu^{2} \mu^{2}$ to equalize all Fermi momenta and gains a pairing energy benefit $\propto \Delta_{0}^{2} \mu^{2}$. As a function of decreasing density, there comes a point (at which $\delta \mu \approx \Delta_{0} / 4$ ) when the system can lower its energy by breaking pairs [3]. Restricting the analysis to spatially homogeneous condensates, the phase that results when CFL Cooper pairs start to break is the gapless CFL phase [3, 4]. However, this phase turns out to be "magnetically unstable" [5, 6, 7], meaning that it is unstable to the formation of counter-propagating currents. If $\Delta_{0}$ is small enough that there is a window of densities for which the gapless CFL phase has a lower free energy than the CFL phase before nuclear matter takes over from quark matter, then some other color superconducting phase(s) with a smaller free energy than the gapless CFL phase must be the ground state of quark matter in this window.

Assuming such a window exists, a possible resolution of the "magnetic instability", in particular for the the lower values of densities, is the crystalline color superconducting phase [8] which is the QCD analogue of a form of non-BCS pairing first considered by Larkin, Ovchinnikov, Fulde and Ferrell (LOFF) [9]. (For two alternate possibilities see [10] and [11] .) This phase is an attractive candidate in the intermediate density regime because it allows pairing to occur even with split Fermi surfaces in the free-energetically optimal way as in the absence of pairing, by permitting Cooper pairs with non-zero net momentum. For example, by allowing $u$ quarks with momentum 
$\mathbf{p}+\mathbf{q}_{\mathbf{3}}$ to pair with $d$ quarks with momentum $-\mathbf{p}+\mathbf{q}_{\mathbf{3}}$, for a fixed $\mathbf{q}_{3}$, we can pair $u$ and $d$ quarks along rings on their respective Fermi surfaces [8, 12]. In coordinate space, this corresponds to a condensate of the form $\langle u d\rangle \sim \Delta_{3} \exp \left(2 i \mathbf{q}_{3} \cdot \mathbf{r}\right)$. The net free energy gained due to pairing is then a balance between increasing $\left|\mathbf{q}_{\mathbf{3}}\right|$ yielding pairing on larger rings while exacting a greater kinetic energy cost. The optimum choice turns out to be $\left|\mathbf{q}_{\mathbf{3}}\right|=\eta \delta \mu_{3}$ with $\eta=1.1997$, corresponding to pairing rings on the Fermi surfaces with opening angle $67.1^{\circ}$ [8]. It is possible to cover larger areas of the Fermi surfaces by allowing Cooper pairs with the same $\left|\mathbf{q}_{3}\right|$ but various $\hat{\mathbf{q}}_{3}$, yielding $\langle u d\rangle \sim \Delta_{3} \sum_{\mathbf{q}_{3}^{a}} \exp \left(2 i \mathbf{q}_{3}^{a} \cdot \mathbf{r}\right)$ with the $\mathbf{q}_{3}^{a}$ chosen from some specified set $\left\{\mathbf{q}_{3}\right\}$. This is a condensate modulated in position space in some crystalline pattern, with the crystal structure defined by $\left\{\mathbf{q}_{3}\right\}$. In this two-flavor context, a Ginzburg-Landau analysis reveals that the best $\left\{\mathbf{q}_{3}\right\}$ contains eight vectors pointing at the corners of a cube, say in the $( \pm 1, \pm 1, \pm 1)$ directions in momentum space, yielding a face-centered cubic structure in position space [12]. In the following section we generalize the pairing ansatz to the three flavor case.

\section{Three flavor crystalline color superconductivity}

In Refs. [13, 14, 15] the possibility that three flavor quark matter has a crystalline color superconducting structure was explored. Considering the pairing of the $u$ and $d$ quarks with the $s$ quark, we use a pairing ansatz of form,

$$
\left\langle\psi_{i \alpha} C \gamma^{5} \psi_{j \beta}\right\rangle \propto \sum_{I} \varepsilon_{I \alpha \beta} \varepsilon_{I i j} \Delta_{I} \sum_{\mathbf{q}_{I}^{a} \in\left\{\mathbf{q}_{I}\right\}} \exp \left(2 i \mathbf{q}_{I}^{a} \cdot \mathbf{r}\right)
$$

This is antisymmetric in color $(\alpha, \beta)$, spin, and flavor $(i, j)$ (where $(1,2,3)$ correspond to $(u, d, s)$ respectively) and is thus a generalization of the CFL condensate to crystalline color superconductivity. For simplicity, we set $\Delta_{1}=0$, neglecting $\langle d s\rangle$ pairing because the $d$ and $s$ Fermi surfaces are twice as far apart from each other as each is from the intervening $u$ Fermi surface. Hence, the index $I$ can be taken to run over 2 and 3 only. $\left\{\mathbf{q}_{2}\right\}\left(\left\{\mathbf{q}_{3}\right\}\right)$ defines the crystal structure of the $\langle u s\rangle$ $(\langle u d\rangle)$ condensate.

We will analyze the system in an NJL model, which gives in the mean field approximation an interaction term

$$
\mathscr{L}_{\text {interaction }}=\frac{1}{2} \bar{\psi} \Delta(\mathbf{r}) \bar{\psi}^{T}+\text { h.c. },
$$

where the proportionality constant in (2.1) is conventionally chosen so that

$$
\Delta(\mathbf{r})=\left(C \gamma^{5}\right) \sum_{I} \varepsilon_{I \alpha \beta} \varepsilon_{I i j} \Delta_{I} \sum_{\mathbf{q}_{I}^{a} \in\left\{\mathbf{q}_{I}\right\}} \exp \left(2 i \mathbf{q}_{I}^{a} \cdot \mathbf{r}\right) .
$$

The authors of [15] calculated the free energy $\Omega$ of several crystalline structures within the weak coupling and Ginzburg-Landau approximations, and found two qualitative rules that guide our understanding of what crystal structures are favored in three flavor quark matter. First, the $\langle u d\rangle$ condensates separately should be chosen to have favorable free energies, as evaluated in the two flavor model of Ref. [12]. Second, the $\langle u d\rangle$ and $\langle u s\rangle$ condensates should be rotated relative to each other in such a way as to maximize the angles between the wave vectors describing the crystal 
structure of the $\langle u d\rangle$ condensate and the antipodes of the wave vectors describing the $\langle u s\rangle$ condensate. This second qualitative rule can be understood as minimizing the "competition" between the two condensates for up quarks on the up Fermi surface [15].

Two of the structures that possess these two features, called the CubeX and $2 \mathrm{Cube} 45 \mathrm{z}$ that we describe below, have a lower $\Omega$ than other considered structures. One or the other of the two, is also favored over unpaired quark matter and the gapless CFL phase over a large parameter range [15, 16], see Fig. 1 .

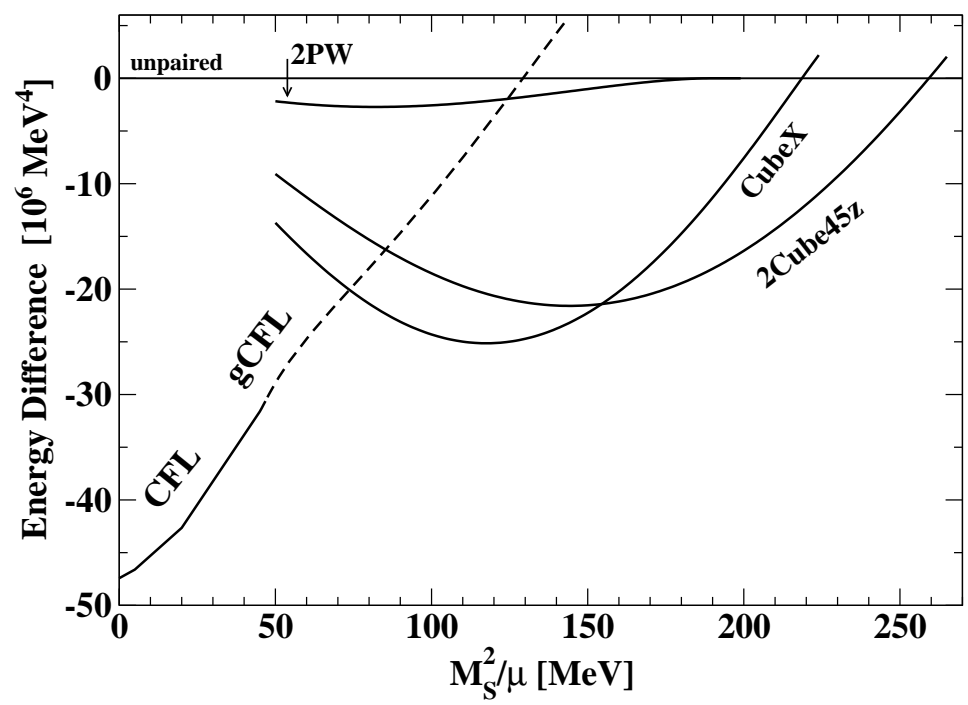

Figure 1: The free energies of CubeX and 2Cube $45 \mathrm{z}$ structures as a function of $M_{s}^{2} / \mu$, which is proportional to the Fermi surface splitting. For comparison we also show the free energy of the CFL phase, of the gapless CFL phase (gCFL) and of a crystalline condensate characterized by two antipodal plane waves (2PW).

Their robustness, however goes with a large gap parameter $\Delta$, and the Ginzburg-Landau expansion parameter $(\Delta / \delta \mu)^{2}$ for these phases can be as large as a fourth for some values of $\mu$, and hence the approximation is at the edge of its validity. Nevertheless, their impressive robustness over a large range of $\mu$ relevant for cores of neutron stars, make them well motivated candidate phases to consider for phenomenological implications. For analysis which go beyond the Ginzburg-Landau approximation see [14, 17].

The CubeX crystal structure consists of eight vectors that belong to two sets of four vectors, $\left\{\hat{\mathbf{q}}_{\mathbf{2}}\right\}$ that can be taken as $\{(1 / \sqrt{3})( \pm \sqrt{2}, 0, \pm 1)\}$, the four possible combinations of the signs giving the four momentum directions, and $\left\{\hat{\mathbf{q}}_{\mathbf{3}}\right\}$ that can be taken as $\{(1 \sqrt{3})(0, \pm \sqrt{2}, \pm 1)\}$. The 2 Cube $45 \mathrm{z}$ crystal structure is specified by two sets of eight unit vectors; the first set $\left\{\hat{\mathbf{q}}_{\mathbf{2}}\right\}$ given by $\{(1 / \sqrt{3})( \pm 1, \pm 1, \pm 1)\}$ and the second one $\left\{\hat{\mathbf{q}}_{\mathbf{3}}\right\}$ given by $\{(1 / \sqrt{3})( \pm \sqrt{2}, 0, \pm 1)\} \cup$ $\{(1 / \sqrt{3})(0, \pm \sqrt{2}, \pm 1)\}$. For these structures, $\left\{\mathbf{q}_{2}\right\}$ can be transformed to $\left\{\mathbf{q}_{3}\right\}$ by rigid rotations, ensuring that there are electrically neutral solutions of the gap equation with $\Delta_{2}=\Delta_{3}=\Delta$ [15], a fact we will use below. 


\section{Phonons}

The crystalline phases of color superconducting quark matter that we have described in the previous Section are unique among all forms of dense matter that may arise within neutron star cores in one respect: they are rigid [18]. They are not solids in the usual sense: the quarks are not fixed in place at the vertices of some crystal structure. Instead, in fact, these phases are superfluid since the condensates all spontaneously break the $U(1)_{B}$ symmetry corresponding to quark number. The diquark condensate, although spatially inhomogeneous, can carry supercurrents [8, 18]. And yet, we shall see that crystalline color superconductors are rigid solids with large shear moduli. It is the spatial modulation of the gap parameter that breaks translation invariance, and it is this pattern of modulation that is rigid. This novel form of rigidity may sound tenuous upon first hearing, but we shall present the effective Lagrangian that describes the phonons in the CubeX and 2Cube $45 \mathrm{z}$ crystalline phases, whose lowest order coefficients have been calculated in the NJL model that we are employing [18]. We shall then extract the shear moduli from the phonon effective action, quantifying the rigidity and indicating the presence of transverse phonons.

Phonons in the crystal correspond to space- and time-varying displacements of the crystalline pattern [19]. In the present context, we introduce displacement fields for the $\langle u d\rangle,\langle u s\rangle$ and $\langle d s\rangle$ condensates by making the replacement

$$
\Delta_{I} \sum_{\mathbf{q}_{I}^{a} \in\left\{\mathbf{q}_{I}\right\}} e^{2 i \mathbf{q}_{I}^{a} \cdot \mathbf{r}} \rightarrow \Delta_{I} \sum_{\mathbf{q}_{I}^{a} \in\left\{\mathbf{q}_{l}\right\}} e^{2 i \mathbf{q}_{I}^{a} \cdot\left(\mathbf{r}-\mathbf{u}_{I}(\mathbf{r})\right)}
$$

in (2.3). One way to obtain the effective action describing the dynamics of the displacement fields $\mathbf{u}_{I}(\mathbf{r})$, including both its form and the values of its coefficients within the NJL model that we are employing, is to take the mean field NJL interaction to be given by (2.2), but with (3.1), and integrate out the fermion fields. Since the gapless fermions do not contribute to the shear modulus, one can integrate out the fermions completely for this calculation. Note that this is not true for the calculation of thermal or transport properties, where the gapless fermions do contribute.

Upon carrying out the fermionic functional integration, we obtain,

$$
\begin{aligned}
& S[\mathbf{u}]=\frac{1}{2} \int d^{4} x \sum_{I} \kappa_{I} \\
& \times\left[\left(\sum_{\mathbf{q}_{I}^{a} \in\left\{\mathbf{q}_{I}\right\}}\left(\hat{q}_{I}^{a}\right)^{m}\left(\hat{q}_{I}^{a}\right)^{n}\right)\left(\partial_{0} u_{I}^{m}\right)\left(\partial_{0} u_{I}^{n}\right)-\left(\sum_{\mathbf{q}_{I}^{a} \in\left\{\mathbf{q}_{I}\right\}}\left(\hat{q}_{I}^{a}\right)^{m}\left(\hat{q}_{I}^{a}\right)^{v}\left(\hat{q}_{I}^{a}\right)^{n}\left(\hat{q}_{I}^{a}\right)^{w}\right)\left(\partial_{\nu} u_{I}^{m}\right)\left(\partial_{w} u_{I}^{n}\right)\right]
\end{aligned}
$$

where $m, n, v$ and $w$ are spatial indices running over $x, y$ and $z$ and where we have defined

$$
\kappa_{I} \equiv \frac{2 \mu^{2}\left|\Delta_{I}\right|^{2} \eta^{2}}{\pi^{2}\left(\eta^{2}-1\right)}
$$

For $\Delta_{1}=0, \Delta_{2}=\Delta_{3}=\Delta$, and $\eta \simeq 1.1997$,

$$
\kappa_{2}=\kappa_{3} \equiv \kappa \simeq 0.664 \mu^{2}\left|\Delta^{2}\right|
$$

$S[\mathbf{u}]$ is the low energy effective action for phonons in any crystalline color superconducting phase, valid to second order in derivatives, to second order in the gap parameters $\Delta_{I}$ and to second 
order in the phonon fields $\mathbf{u}_{I}$. Because we are interested in long wavelength, small amplitude, phonon excitations, expanding to second order in derivatives and in the phonon fields is satisfactory. The Ginzburg-Landau expansion, which gives a series in $(\Delta / \delta \mu)^{2}$, is not under quantitative control for these most favorable phases, as we discussed in the previous Section. But as we shall see, for glitch phenomenology, the main requirement from the shear modulus calculation is that it should be large, and given that we get much larger values than those obtained for conventional neutros star crusts, there is at present no great motivation to go to higher orders. At this order in $\left(\Delta_{I}\right)^{2}$, there is no mixing between different $\mathbf{u}_{I}$, and they can all be treated independently.

In order to extract the shear moduli, we need to compare the phonon effective action to the theory of elastic media [20], which requires introducing the strain tensor

$$
s_{I}^{m v} \equiv \frac{1}{2}\left(\frac{\partial u_{I}^{m}}{\partial x^{v}}+\frac{\partial u_{I}^{v}}{\partial x^{m}}\right) .
$$

We then wish to compare the action (3.2) to

$$
S[\mathbf{u}]=\frac{1}{2} \int d^{4} x\left(\sum_{I} \sum_{m} \rho_{I}^{m}\left(\partial_{0} u_{I}^{m}\right)\left(\partial_{0} u_{I}^{m}\right)-\sum_{I} \sum_{\substack{m n \\ v w}} \lambda_{I}^{m v n w} s_{I}^{m v} s_{I}^{n w}\right),
$$

which is the general form of the action for $\mathbf{u}_{I}$ that don't mix, in the case in which the effective action is quadratic in displacements and which defines the elastic modulus tensor $\lambda_{I}^{m v n w}$ for this case. In this case, the stress tensor (in general the derivative of the potential energy with respect to $\left.s_{I}^{m v}\right)$ is given by

$$
\sigma_{I}^{m v}=\lambda_{I}^{m v n w} s_{I}^{n w}
$$

The diagonal components of $\sigma$ are proportional to the compression exerted on the system and are therefore related to the bulk modulus of the crystalline color superconducting quark matter. Since unpaired quark matter has a pressure $\sim \mu^{4}$, it gives a contribution to the bulk modulus that completely overwhelms the contribution from the condensation into a crystalline phase, which is of order $\mu^{2} \Delta^{2}$. We shall therefore not calculate the bulk modulus. On the other hand, the response to shear stress arises only because of the presence of the crystalline condensate. The shear modulus is defined as follows. Imagine exerting a static external stress $\sigma_{I}$ having only an off-diagonal component, meaning $\sigma_{I}^{m v} \neq 0$ for a pair of space directions $m \neq v$, and all the other components of $\sigma$ are zero. The system will respond with a strain $s_{I}^{n w}$. The shear modulus in the $m v$ plane is then

$$
v_{I}^{m v} \equiv \frac{\sigma_{I}^{m v}}{2 s_{I}^{m v}}=\frac{1}{2} \lambda_{I}^{m v m v},
$$

where the indices $m$ and $v$ are not summed. For a general quadratic potential with $\sigma_{I}^{m v}$ given by (3.7), $v_{I}^{m v}$ simplifies partially but the full simplification given by the last equality in (3.8) only arises for special cases in which the only nonzero entries in $\lambda^{m v n w}$ with $m \neq v$ are the $\lambda^{m v m v}$ entries, as is the case for all the crystal structures that we consider.

For a given crystal structure, upon evaluating the sums in (3.2) and then using the definition (3.5) to compare (3.2) to (3.6), we can extract expressions for the $\lambda$ tensor and thence for the shear moduli. This analysis, described in detail in [18], shows that in the CubeX phase

$$
v_{2}=\frac{16}{9} \kappa\left(\begin{array}{lll}
0 & 0 & 1 \\
0 & 0 & 0 \\
1 & 0 & 0
\end{array}\right), \quad v_{3}=\frac{16}{9} \kappa\left(\begin{array}{lll}
0 & 0 & 0 \\
0 & 0 & 1 \\
0 & 1 & 0
\end{array}\right)
$$


while in the 2 Cube $45 \mathrm{z}$ phase

$$
v_{2}=\frac{16}{9} \kappa\left(\begin{array}{lll}
0 & 1 & 1 \\
1 & 0 & 1 \\
1 & 1 & 0
\end{array}\right), v_{3}=\frac{16}{9} \kappa\left(\begin{array}{lll}
0 & 0 & 1 \\
0 & 0 & 1 \\
1 & 1 & 0
\end{array}\right) .
$$

We shall see in the next Section that it is relevant for glitch phenomenology to check that both these crystals have enough nonzero entries in their shear moduli $v_{I}$ that if there are rotational vortices are pinned within them, a force seeking to move such a vortex is opposed by the rigidity of the crystal structure described by one or more of the nonzero entries in the $v_{I}$. This is demonstrated in [18].

We see that all the nonzero shear moduli of both the CubeX and 2Cube $45 \mathrm{z}$ crystalline color superconducting phases turn out to take on the same value,

$$
v_{\mathrm{CQM}}=\frac{16}{9} \kappa=1.18 \mu^{2} \Delta^{2}=2.47 \frac{\mathrm{MeV}}{\mathrm{fm}^{3}}\left(\frac{\Delta}{10 \mathrm{MeV}}\right)^{2}\left(\frac{\mu}{400 \mathrm{MeV}}\right)^{2},
$$

where $\mu$ is expected to lie between 350 to $500 \mathrm{MeV}$ and $\Delta$ may be taken to be between 5 and $25 \mathrm{MeV}$ to obtain numerical estimates.

From (3.11) we first of all see that the shear modulus is in no way suppressed relative to the scale $\mu^{2} \Delta^{2}$ that could have been guessed on dimensional grounds. And, second, we discover that a quark matter core in a crystalline color superconducting phase is 20 to 1000 times more rigid than the crust of a conventional neutron star [21]. Finally, one can extract the phonon dispersion relations from the effective action (3.2). The transverse phonons, whose restoring force is provided by the shear modulus turn out to have direction-dependent velocities that are typically a substantial fraction of the speed of light, in the specific instances evaluated in [18] being given by $\sqrt{1 / 3}$ and $\sqrt{2 / 3}$. This is yet a third way of seeing that this superfluid phase of matter is rigid indeed.

\section{Rigid quark matter}

The existence of a rigid crystalline color superconducting core within neutron stars may have a variety of observable consequences. For example, if some agency (e.g. magnetic fields not aligned with the rotation axis) could maintain the rigid core in a shape that has a nonzero quadrupole moment, gravity waves would be emitted. The LIGO non-detection of such gravity waves from nearby neutron stars already limits the possibility that they have rigid cores that are deformed to the maximum extent allowed by the shear modulus (3.11) [22, 23, 24]. Perhaps the most exciting implication of a rigid core, however, is the possibility that (some) pulsar "glitches" could originate deep within a neutron star, in its quark matter core.

A spinning neutron star observed as a pulsar gradually spins down as it loses rotational energy to electromagnetic radiation. But, every once in a while the angular velocity at the crust of the star is observed to increase suddenly in a dramatic event called a glitch. The standard explanation [25] (see [18] for more Refs.) requires the presence of a superfluid in some region of the star which also features a rigid structure that can pin the vortices in the rotating superfluid and that does not easily deform when the vortices pinned to it are under tension.

As a spinning pulsar slowly loses angular momentum over years, since the angular momentum of any superfluid component of the star is proportional to the density of vortices, the vortices "want" 
to move apart. However, if the vortices are pinned to a rigid structure, these vortices do not move and after a time this superfluid component of the star is spinning faster than the rest of the star. When the "tension" built up in the array of pinned vortices reaches a critical value, there is a sudden "avalanche" in which vortices unpin, move outwards reducing the angular momentum of the superfluid, and then re-pin. As this superfluid suddenly loses angular momentum, the rest of the star, including in particular the surface whose angular velocity is observed, speeds up - a glitch. In the standard explanation of pulsar glitches, this occurs in the inner crust of a neutron star where a neutron superfluid coexists with a rigid array of positively charged nuclei that may serve as vortex pinning sites. In recent work, Link has concluded that this scenario is not viable because once neutron vortices are moving through the inner crust, as must happen during a glitch, they are so resistant to bending that they can never re-pin [26]. Link concludes that we do not currently understand the origin of glitches as a crustal phenomenon.

By virtue of being simultaneously superfluids and rigid solids, the crystalline phases of quark matter provide all the necessary conditions to be the locus in which (some) pulsar glitches originate. Their shear moduli (3.11), makes them more than rigid enough for glitches to originate within them. The crystalline phases are at the same time superfluid, and it is reasonable to expect that the superfluid vortices will have lower free energy if they are centered along the intersections of the nodal planes of the underlying crystal structure, i.e. along lines along which the condensate already vanishes even in the absence of a rotational vortex. A crude estimate of the pinning force on vortices within crystalline color superconducting quark matter indicates that it is sufficient [18]. So, the basic requirements for superfluid vortices pinning to a rigid structure are all present. The central questions that remain to be addressed are the explicit construction of vortices in the crystalline phase and the calculation of their pinning force, as well as the calculation of the timescale over which sudden changes in the angular momentum of the core are communicated to the (observed) surface, presumably either via the common electron fluid or via magnetic stresses.

Much theoretical work remains before the hypothesis that pulsar glitches originate within a crystalline color superconducting neutron star core is developed fully enough to allow it to confront data on the magnitudes, relaxation timescales, and repeat rates that characterize the data. Nevertheless, this hypothesis offers one immediate advantage over the conventional scenario that relied on vortex pinning in the neutron star crust. Link has observed that it is impossible for a neutron star anywhere within which rotational vortices are pinned to precess [27], and yet there is now evidence that several pulsars are precessing [28]. Since all neutron stars have crusts, the precession of any pulsar is inconsistent with the pinning of vortices within the crust, a requirement in the standard explanation of glitches. On the other hand, perhaps not all neutron stars have crystalline quark matter cores - for example, perhaps the lightest neutron stars have nuclear matter cores. Then, if vortices are never pinned in the crust but are pinned within a crystalline quark matter core, those neutron stars that do have a crystalline quark matter core can glitch but cannot precess while those that don't can precess but cannot glitch.

\section{Acknowledgements}

Massimo Mannarelli and Rishi Sharma would like to thank Krishna Rajagopal for collaboration on the work presented here. They would also like to thank the organizers of the conference 
"Confienement 2008" for giving us an opportunity to present our work. MM has been supported by the "Bruno Rossi" fellowship program and by the Spanish grant AYA 2005-08013-C03-02. This research was supported in part by the Office of Nuclear Physics of the Office of Science of the U.S. DoE, contract \#DE-AC02-05CH11231, cooperative research agreement \#DF-FC0294ER40818 and LANS, LLC for the NNSA of the DoE, contract \#DE-AC52-06NA25396.

\section{References}

[1] For reviews, see K. Rajagopal and F. Wilczek, arXiv:hep-ph/0011333; M. G. Alford, A. Schmitt, K. Rajagopal and T. Schafer, Rev. Mod. Phys. 80, 1455 (2008) [arXiv:0709.4635 [hep-ph]]; M. Mannarelli, arXiv:0812.2831 [hep-ph].

[2] M. G. Alford, K. Rajagopal and F. Wilczek, Nucl. Phys. B 537, 443 (1999) [arXiv:hep-ph/9804403].

[3] M. Alford, C. Kouvaris and K. Rajagopal, Phys. Rev. Lett. 92, 222001 (2004) [arXiv:hep-ph/0311286].

[4] K. Fukushima, C. Kouvaris and K. Rajagopal, Phys. Rev. D 71, 034002 (2005) [arXiv:hep-ph/0408322].

[5] M. Huang and I. A. Shovkovy, Phys. Rev. D 70, 051501 (2004) [arXiv:hep-ph/0407049].

[6] R. Casalbuoni, R. Gatto, M. Mannarelli, G. Nardulli and M. Ruggieri, Phys. Lett. B 605, 362 (2005) [Erratum-ibid. B 615, 297 (2005)] [arXiv:hep-ph/0410401].

[7] K. Fukushima and K. Iida, Phys. Rev. D 76, 054004 (2007) [arXiv:0705.0792 [hep-ph]].

[8] M. G. Alford, J. A. Bowers and K. Rajagopal, Phys. Rev. D 63, 074016 (2001) [arXiv:hep-ph/0008208].

[9] A. I. Larkin and Yu. N. Ovchinnikov, Zh. Eksp. Teor. Fiz. 47, 1136 (1964)[Sov. Phys. JETP 20, 762 (1965)]; P. Fulde and R. A. Ferrell, Phys. Rev. 135, A550 (1964); S. Takada and T. Izuyama, Prog. Theor. Phys. 41, 635 (1969).

[10] A. Kryjevski, Phys. Rev. D 77, 014018 (2008), [arXiv:hep-ph/0508180]; T. Schafer, Phys. Rev. Lett. 96, 012305 (2006) [arXiv:hep-ph/0508190].

[11] E. V. Gorbar, M. Hashimoto and V. A. Miransky, Phys. Lett. B 632, 305 (2006) [arXiv:hep-ph/0507303].

[12] J. A. Bowers and K. Rajagopal, Phys. Rev. D 66, 065002 (2002) [arXiv:hep-ph/0204079].

[13] R. Casalbuoni, R. Gatto, N. Ippolito, G. Nardulli and M. Ruggieri, Phys. Lett. B 627, 89 (2005).

[14] M. Mannarelli, K. Rajagopal and R. Sharma, Phys. Rev. D 73, 114012 (2006).

[15] K. Rajagopal and R. Sharma, Phys. Rev. D 74, 094019 (2006) [arXiv:hep-ph/0605316]; K. Rajagopal and R. Sharma, J. Phys. G 32, S483 (2006) [arXiv:hep-ph/0606066].

[16] R. Casalbuoni, M. Ciminale, R. Gatto, G. Nardulli and M. Ruggieri, Phys. Lett. B 642, 350 (2006) [arXiv:hep-ph/0606242].

[17] D. Nickel and M. Buballa, arXiv:0811.2400 [hep-ph].

[18] M. Mannarelli, K. Rajagopal and R. Sharma, Phys. Rev. D 76, 074026 (2007) [arXiv:hep-ph/0702021]. 
[19] R. Casalbuoni, E. Fabiano, R. Gatto, M. Mannarelli and G. Nardulli, Phys. Rev. D 66, 094006 (2002) [arXiv:hep-ph/0208121].

[20] L. D. Landau and E. M. Lifschitz, Theory of Elasticity, 3rd edition, Oxford, Pergamon (1981).

[21] T. Strohmayer, H. M. van Horn, S. Ogata, H. Iyetomi and S. Ichimaru, Astrophys. J. 375, 679 (1991).

[22] B. Abbott et al. [LIGO Scientific Collaboration], Phys. Rev. D 76, 042001 (2007) [arXiv:gr-qc/0702039].

[23] B. Haskell, N. Andersson, D. I. Jones and L. Samuelsson, Phys. Rev. Lett. 99, 231101 (2007) [arXiv:0708.2984 [gr-qc]].

[24] L. M. Lin, Phys. Rev. D 76, 081502(R) (2007), arXiv:0708.2965 [astro-ph]. The analogous calculation for a star that is rigid throughout was done in B. J. Owen, Phys. Rev. Lett. 95, 211101 (2005) [arXiv:astro-ph/0503399].

[25] P. W. Anderson and N. Itoh, Nature 256, 25 (1975). See [18] for references to subsequent literature.

[26] B. Link, talk given at INT Workshop on The Neutron Star Crust and Surface, Seattle (2007) and private communication.

[27] T. Akgün, B. Link and I. Wasserman, Mon. Not. of the Royal Astro. Soc. 365 (2), 653-672 (2006).

[28] I. H. Stairs, A. G. Lyne and S. L. Shemar, Nature 406, 484-6 (2000, Aug 3); T. V. Shabanova, A. G. Lyne and J. O. Urama, The Astrophys. Journal, 552, 321-325, (2001, May 1); A. E. Chukwude, A. A. Ubachukwu and P. N. Okeke, Astron. Astrophys. 399, 231 (2003). 